\title{
HDAC4 in ischemic stroke: mechanisms and therapeutic potential
}

\author{
Qingsheng Kong ${ }^{1,2 \dagger}$, Yongnan $\mathrm{Hao}^{3 \dagger}$, Xin $\mathrm{Li}^{4 \dagger}$, Xin Wang ${ }^{5,6}$, Bingyuan $\mathrm{Ji}^{5^{*}}$ and Yili $\mathrm{Wu}^{1,5,6^{*}}$ (D)
}

\begin{abstract}
Stroke is one of the leading causes of death and disability worldwide, and the majority of the cases are ischemic stroke. However, it still lacks effective treatment except for thrombolytic therapy in an extremely narrow time window. Increased evidence suggests that histone deacetylase 4 (HDAC4) was dysregulated in ischemic stroke, which plays a key role in the pathogenesis of ischemic stroke and post-stroke recovery by affecting neuronal death, angiogenesis, and neurogenesis. Therefore, we aim to review the dysregulation of HDAC4 in ischemic stroke and the role of dysregulated HDAC4 in the pathogenesis of ischemic stroke. Furthermore, the therapeutic potential of modulating HDAC4 in ischemic stroke is discussed.
\end{abstract}

Keywords: HDAC4, Ischemic stroke, Cell death, Angiogenesis, Neurogenesis

\section{Background}

Stroke is one of the leading causes of death and disability worldwide [1]. In the USA, it is the leading cause of long-term disability, including both physical and cognitive deficits, while it is the leading cause of death in China $[2,3]$. The prevalence of stroke continues increasing, and the direct medical costs will reach $\$ 184.1$ billion in the USA by 2030. In addition, increased risk of neurodegenerative diseases, such as Alzheimer's disease, was observed in patients who experienced a stroke, which further increases the burden of health care [4]. Ischemic stroke is the major subtype of stroke, accounting for $87 \%$ of stroke cases. However, current treatments for ischemic stroke are only limited to thrombolytic therapy within an extremely narrow time window [5]. Thus, developing novel therapeutic approaches for ischemic stroke is urgent.

Histone deacetylases (HDACs) along with histone acetyltransferases (HATs) regulate chromatin remodeling and subsequent gene transcription by controlling the status of histone acetylation. Compared with histone acetylation,

\footnotetext{
*Correspondence: jby2006@126.com; yili_wu2004@yahoo.ca; wuyili@mail.jnmc.edu.cn

${ }^{\dagger}$ Qingsheng Kong, Yongnan Hao and Xin Li contributed equally to this work. ${ }^{5}$ Institute of Mental Health, Jining Medical University, 133 Hehua Road, Taibaihu New District, Jining 272067, Shandong, China

${ }^{1}$ Collaborative Innovation Center for Birth Defect Research and Transformation of Shandong Province, Jining Medical University, 133 Hehua Road, Taibaihu New District, Jining 272067, Shandong, China Full list of author information is available at the end of the article
}

histone deacetylation induces a condensed chromatin conformation, contributing to the repression of gene transcription which is involved in diverse physiological processes. Moreover, the function of HDACs is not limited to the histone deacetylation. Recent evidence suggests that HDACs may also contribute to the deacetylation of non-histone proteins [6]. In addition, HDACs also have deacetylase-independent functions, including other modifications of histone, such as methylation [6-8]. Importantly, HDACs are dysregulated in a number of brain disorders, which is implicated in the pathogenesis of these diseases, e.g., ischemic stroke, autism, Alzheimer's disease, and depressive disorders [9-15]. It suggests that HDACs might be potential targets for the treatment of brain disorders.

Growing evidence indicates that HDAC4 is a specific target for the treatment of ischemic stroke. First, dysregulated HDAC4 was observed in ischemic stroke, which does play a key role in the pathogenesis of ischemic stroke and post-stroke recovery by affecting neuronal death, angiogenesis, and neurogenesis [16-20]. For example, HDAC4 is reduced in ischemic stroke model animals and oxygen-glucose deprivation (OGD)-treated neurons, while increased HDAC4 expression reduces infarct volume in ischemic stroke model animals and increases cell viability of OGD-treated neuronal cells $[9,10$, $21,22]$. In addition, HDAC4 has a significant effect on a cognitive function which could be impaired by ischemic stroke [23]. For example, conditional deletion of HDAC4 
leads to learning and memory deficits [24-26]. It indicates that HDAC4 might be a target for the treatment of ischemic stroke. Therefore, we aim to review the dysregulation of HDAC4 in ischemic stroke and the role of HDAC4 in the pathogenesis of ischemic stroke and post-stroke recovery. Furthermore, the therapeutic potential of modulating HDAC4 in ischemic stroke is discussed.

\section{Mechanisms of ischemic stroke and post-stroke recovery \\ Cell death and synaptic impairment}

Depending on the severity of reduced blood supply, acute and delayed cell death, i.e., necrosis and apoptosis, occurs in the core region and penumbra region of the ischemic territory, respectively [27]. Necrosis occurs within minutes after stroke, which cannot be rescued. However, apoptosis and impaired synaptic function in the penumbra could be salvageable by proper interventions, suggesting that preventing apoptosis and recovering synaptic function in the penumbra region may be an effective approach to improve post-stroke recovery. Ischemia/reperfusion injury-induced apoptosis and synaptic impairment in the penumbra are mediated by a number of mechanisms, including excitotoxicity, oxidative stress, inflammatory response, and endoplasmic reticulum (ER) stress [28-30]. For example, the dysregulation of synaptic proteins, e.g., subunits of $N$-methyl-D-aspartic acid (NMDA) receptors, was observed in ischemic stroke, which not only led to synaptic dysfunction but also contributed to excitotoxic cell death [30]. It suggests that suppressing detrimental pathways may have therapeutic potential for ischemic stroke by protecting the penumbra from neuronal death and synaptic impairment.

\section{Angiogenesis}

During an acute ischemic stroke, the reduction of blood supply in the ischemic area often activates angiogenesis, a neurovascular remodeling process, which is a compensatory response to the reduction of oxygen. Numerous studies have shown that angiogenesis is positively correlated with the survival rate of patients who experienced an ischemic stroke, indicating that angiogenesis is an endogenous brain repair mechanism [31-33]. Thus, the modulation of vascular growth in the ischemic area could be a therapeutic approach for ischemic stroke. Indeed, the beneficial effects of direct injections or gene transfer of angiogenic factors have been demonstrated by inducing therapeutic angiogenesis in ischemic stroke, myocardial infarction, and limb ischemic injury [34-36]. Enhanced angiogenesis is not only beneficial to the cell survival in the penumbra region but also promotes neurogenesis facilitating post-stroke recovery, which orchestrates post-stroke recovery [37].

\section{Neurogenesis}

Neurogenesis, including neural stem cell proliferation, migration, and differentiation, plays a key role in the chronic stage of post-stroke recovery [38]. Increased stem cell proliferation was observed in post-stroke patients and mice model. However, the majority of newly born cells die during the first 2 weeks after their formation. It suggests that improving the survival, migration, and differentiation of newly formed cells is the key of enhancing post-stroke neurogenesis. In addition, repetitive transcranial magnetic stimulation ameliorates cognitive impairment by enhancing neurogenesis in rats with ischemic stroke [39]. Moreover, the consistent efficacy of two approaches, stem cell transplantation and stimulating endogenous neurogenesis, was observed in animal models of ischemic stroke [40, 41]. However, the therapeutic effect of transplantation of stem cell for ischemic stroke needs to be further investigated, and clinical trials are still ongoing [40-42].

\section{Characteristics of HDAC4}

HDACs are a large family of enzymes, regulating chromatin remodeling and subsequent gene transcription mainly by controlling the status of histone acetylation. According to the sequence homology, HDACs are grouped into class I (HDAC1, 2, 3, and 8), class II (IIa: HDAC4, 5, 7, and 9; IIb: HDAC6 and 10), class III (SIRT1-7), and class IV (HDAC11). The HDAC4 protein consists of a long $\mathrm{N}$-terminal domain and a highly conserved C-terminal catalytic domain [15]. Compared with most of HDACs, HDAC4 is usually trapped in the cytoplasm. Its shuttling between the cytoplasm and nucleus is tightly controlled by both the phosphorylation status of HDAC4 and its interacting partners, such as calcium/calmodulin-dependent kinase II (CaMKII), protein phosphotase 2A (PP2A), protein kinase $\mathrm{C}$ (PKC), and tyrosine 3-monooxygenase/tryptophan 5-monooxygenase activation protein (14-3-3) [43-46]. For example, HDAC4 is the substrate of CaMKII, which can export HDAC4 to the cytoplasm [47].

Compared with other HDACs, HDAC4 per se features weak histone deacetylase activity. It may also contribute to the histone deacetylation via interacting with HDAC3 and HDAC5, respectively [48-50]. Moreover, HDAC4 does have histone deacetylase-independent functions. For example, HDAC4 is involved in histone methylation contributing to the regulation of gene transcription [8]. In addition, HDAC4 could regulate gene transcription by interacting with multiple transcriptional factors, including runt-related transcription factor 2 (Runx2), myocyte enhancer factor 2 (MEF2), serum response factor (SRF), heterochromatin protein 1(HP1), nuclear factor kappa $B(\mathrm{NF}-\kappa \mathrm{B})$, and activating transcription factor 4 (ATF4). [51-53]. Furthermore, HDAC4 is implicated in regulating protein SUMOylation by interacting 
with the SUMO-conjugating enzyme Ubc9 (Ubc9) [54]. Thus, HDAC4 may contribute to a number of physiological and pathological processes via histone deacetylase-dependent and deacetylase-independent pathways $[51,52]$.

\section{Dysregulation of HDAC4 in ischemic stroke}

HDAC4 is highly expressed in the brain, mainly in neurons [9]. Recent studies indicate that HDAC4 is dysregulated in ischemic stroke, which may play a pivotal role in the pathogenesis of ischemic stroke and post-stroke recovery. Compared with sham treatment, middle cerebral artery occlusion (MCAO)/reperfusion significantly reduces the expression of HDAC4 in the cortex of rats, which is mediated by NADPH oxidase $[9,10]$. Consistently, the HDAC4 expression is significantly reduced in the cardiomyocytes following ischemia/reperfusion injury [55]. However, the expression of HDAC4 is increased in oligodendrocyte progenitor cells in the brains of ischemic stroke model rats [56].

A number of microRNAs targeting HDAC4 were altered in ischemic stroke, which may also contribute to the dysregulation of HDAC4 in ischemic stroke. For example, miR-9 and miR-124 are markedly increased in both serum and CSF of patients with ischemic stroke $[57,58]$. However, Liu et al. showed that serum miR-124 and miR-9 were reduced in patients with ischemic stroke, although the sample size was small [59]. In addition, the reduction of miR-9 was detected in the brain of ischemic stroke model mice [60]. Moreover, miR-206 and miR-29b, two microRNAs targeting HDAC4, are significantly increased in ischemic rat brains and in OGD-treated primary neurons [61-63]. It suggests that the combination effect of dysregulated microRNAs may contribute to the reduction of HDAC4 in ischemic stroke.

In addition to HDAC4 expression, nuclear shuttling of HDAC4 is altered in ischemic stroke, which plays an important role in the pathogenesis of stroke and poststroke recovery. Increased HDAC4 nuclear shuttling was observed in the neurons of ischemic stroke model mice/ ats and in oxygen-glucose deprivation (OGD)-treated neurons, while the overexpression of calcium/calmodulindependent protein kinase IV (CaMKIV) reduced the levels of nuclear HDAC4 in ischemic stroke [11, 64]. However, increased cytoplasmic HDAC4 expression was detected in oligodendrocyte progenitor cells in the brains of ischemic stroke model rats [56].

\section{The role of HDAC4 in ischemic stroke and underlying mechanisms}

HDAC4 in neuronal death and synaptic impairment

Accumulated evidence indicates that HDAC4 plays an important role in the post-stroke recovery by modulating neuronal death and synaptic plasticity (Fig. 1). First, HDAC4 deficiency causes a progressive loss of neurons in the cerebellum of mice, while the forcing expression of HDAC4 protects neurons from cell death [16]. Moreover, the HDAC4-C-terminal fragment is crucial to rescue HDAC4 knockdown-induced cell death and a reduction of synaptic strength in mouse brains [51]. Zhang et al. showed that reduced HDAC4 expression is associated with blood-brain barrier (BBB) breakdown contributing to ischemia/reperfusion injury-induced infarct in ischemic stroke model rats, while increased HDAC4 expression ameliorates BBB injury, contributing to the reduced infarct volume [10]. Consistently, class IIa histone deacetylase-specific inhibitor increases mortality and infarct volume in the brains of ischemic stroke model rats and exacerbates neuronal remodeling impairment, such as reduced dendritic and axonal and myelination densities [65]. However, pan-HDAC inhibitors have a protective effect on stroke [66, 67]. Moreover, HDAC4 increases cell viability of OGD-treated cells via reducing high-mobility group protein 1(HMGB1) expression [9]. In addition, a proteomics analysis indicated that HDAC4 is a regulator

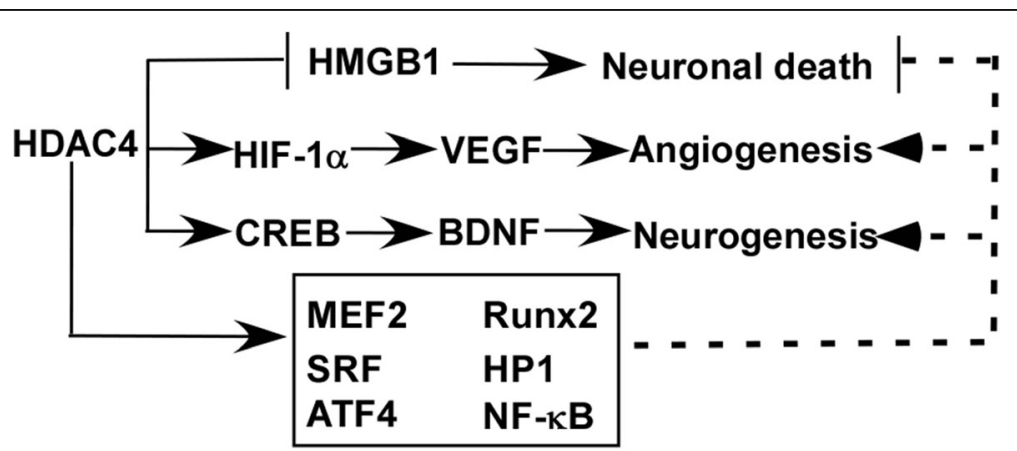

Fig. 1 The role of HDAC4 in ischemic stroke and underlying mechanisms. HDAC4 could inhibit neuronal death via reducing HDMGB1expression and release, while it promotes angiogenesis and neurogenesis via HIF-1a-VEGF signaling and CREB-BDBF signaling, respectively. The interacting partners of HDAC4, MEF2, Runx2, SRF, HP1, ATF4, F-KB, etc. might also mediate its role in the neuronal death, angiogenesis, and neurogenesis in ischemic stroke. The solid line represents known mechanisms, while the dash line represents possible mechanisms 
of proteins involved in neuronal excitability and synaptic plasticity [68]. Silencing HDAC4 expression results in the impairment of synaptic plasticity and learning and memory deficits in both mice and Drosophila, although one report showed that HDAC4 knockdown with siRNA improved the survival of OGD-treated neurons [11, 26, 69]. Currently, mechanisms of reduced HDAC4 in ische$\mathrm{mia} /$ reperfusion injury-induced neuronal death and synaptic impairment remain elusive. However, a number of studies indicate that the effect of HDAC4 on neuronal death and synaptic impairment might be mediated by its partners, e.g., Runx2, MEF2, SRF, HP1, NF-kB, and ATF4, contributing to the processes of ER stress, inflammation, and oxidative stress response [16, 51-53, 70, 71]. For example, HDAC4 overexpression causes ATF4 retention in the cytoplasm, inhibiting ER stress-induced apoptosis, while HDAC4 reduction exacerbates ER stress-induced apoptosis [53].

In addition to HDAC4 levels, nuclear shuttling of HDAC4 also contributes to neuronal death and synaptic impairment in ischemic stroke. Nuclear HDAC4 represses the expression of constituents of synapses leading to the impairment of synaptic architecture and strength in mice [51]. In addition, the neuroprotective effect of CaMKIV on OGD neurons is mediated by reducing nuclear HDAC4 [11]. Moreover, mice carrying nuclear HDAC4 mutant exhibit deficits in neurotransmission, learning, and memory [51]. Yuan et al. reported that ischemic stroke-induced nuclear shuttling of HDAC4 strongly facilitated OGD-induced neuronal death and exacerbated infarct volume and functional deficits in ischemic model mice [11]. In addition, accumulation of nuclear HDAC4 exerts neurotoxicity in models of Parkinson's disease [72].

\section{HDAC4 in angiogenesis}

Post-stroke angiogenesis has a beneficial effect on cell survival and stroke recovery. Qian et al. reported that siRNA-induced HDAC4 reduction suppressed hypoxiainducible factor- $1 \alpha$ (HIF-1 $\alpha)$ expression, which inhibited HIF-1 $\alpha$-associated vascular endothelial growth factor (VEGF) expression in ischemia/reperfusion injury [73, 74]. It suggests that HDAC4 alteration may regulate the angiogenesis in ischemic stroke via HIF- $1 \alpha$-VEGF signaling (Fig. 1). Moreover, HDAC4 phosphorylation is also the key regulator of angiogenesis. Phosphorylation of HDAC4 is remarkably upregulated in the endothelial cells under hypoxic conditions while blocking the phosphorylation of HDAC4 inhibits endothelial cell migration and tube formation, which is associated with the suppression of HIF- $1 \alpha-V E G F$ signaling [75]. Consistently, Liu et al. showed that phosphorylation of HDAC4 was associated with the induction of HIF-1 $\alpha$-VEGF signaling, promoting angiogenesis in ischemic stroke model mice and cells [75]. GO6976, an inhibitor of HDAC4, blocks the phosphorylation of HDAC4 and inhibits the tube formation and migration of endothelial cells [75]. It suggests that HDAC4 phosphorylation facilitates angiogenesis in ischemic stroke. Moreover, HDAC4 may be involved in angiogenesis via its interacting partners, such as NF- $k B$ [76]. Furthermore, Madelaine et al. identified miR-9 inhibition as a positive regulator of neurogenesis and angiogenesis [77]. As HDAC4 is a target of miR-9, it may contribute to the effect of miRNA-9 inhibition on angiogenesis and neurogenesis, suggesting that HDAC4 might be a potential target for the treatment of ischemic stroke.

\section{HDAC4 in neurogenesis}

Growing evidence indicates that HDAC4 may contribute to neurogenesis via regulating the expression and function of multiple molecules. First, HDAC4 regulates the activity and expression of cAMP response element-binding protein (CREB) and brain-derived neurotrophic factor (BDNF), respectively, which play a key role in neurogenesis after ischemic stroke [18-20, 78] (Fig. 1). For example, increased CREB activity and BDNF expression promote post-ischemic stroke neurogenesis and neuroregeneration in rats. However, nuclear shuttling of HDAC4 suppresses the transcriptional activity of CREB by reducing the interaction among acetyltransferase, CBP, and CREB, leading to the reduction of BDNF. [18-20]. In addition, HDAC4 might be another key mediator of the effect of miRNA-9 on neurogenesis in ischemic stroke as HDAC4 is the target of miRNA-9 [77, 79]. Moreover, HDAC4 may be implicated in neurogenesis by regulating the activity of its partners, such as Runx2, MEF2, SRF, HP1, NF-kB, and ATF4. For example, MEF2 promotes neurogenesis while nuclear HDAC4 suppresses the activity of MEF2 [79-81]. The above evidence suggests that the alteration of HDAC4 expression and nuclear shuttling in ischemic stroke may play a pivotal role in post-stroke recovery by affecting neurogenesis.

\section{Clinical perspectives}

HDAC4, a unique target for ischemic stroke treatment

HDAC4 is a unique target for the treatment of ischemic stroke compared with other HDACs, such as HDAC2 [16-20]. For example, HDAC4 features different characteristics and plays an opposite role in ischemic stroke compared with HDAC2 (Table 1). HDAC4 and HDAC2 genes are located at chromosome $2 \mathrm{q} 37$ and chromosome 6q21, respectively, encoding1084 and 488 amino acids, respectively. HDAC4 contains both intrinsic nuclear localization signal and nuclear export signal, while HDAC2 only contains a nuclear localization signal [82-84]. Thus, HDAC2 is mainly localized in the nucleus, while 
Table 1 Difference between HDAC4 and HDAC2

\begin{tabular}{lll}
\hline & HDAC4 & HDAC2 \\
\hline Features & & \\
Gene locus (chromosome) & $2 \mathrm{q} 37$ & $6 \mathrm{q} 21$ \\
Number of amino acids & 1084 & 488 \\
Nuclear localization signal & + & + \\
Nuclear export signal & + & - \\
Subcellular distribution & Cytoplasm/ & Nucleus \\
& nucleus & Strong \\
Histone deacetylase activity & Weak & Impaired \\
Effect on cognitive function & Beneficial & \\
Ischemic stroke & & Increased \\
Altered expression & Reduced & - \\
Altered distribution & Increased nuclear & \\
& shuttling & \\
Rescue effect of class-specific & - & Increased \\
inhibitor on neurological deficits & & Reduced \\
Effect on infarct size & &
\end{tabular}

HDAC4 enriches in the cytoplasm and shuttles between the cytoplasm and nucleus [82-84]. Compared with HDAC2, HDAC4 per se features weak histone deacetylase activity as the critical tyrosine residue within the catalytic domain is substituted by histidine [85]. Compared with HDAC2, HDAC4 interacts with multiple partners, e.g., Runx, MEF2, SRF, HP1, NF-кB, 14-3-3, and Ubc9 [16, 5153, 70, 71]. HDAC4's partners may mediate HDAC4' function in ischemic stroke as the partners are involved in the key processes of ischemic stroke, i.e., neuronal death, angiogenesis, and neurogenesis [53, 76, 79-81] (Fig. 1). Conditional deletion of HDAC4 leads to learning and memory deficits, while global HDACs inhibitors or HDAC2 reduction significantly improves learning and memory function in mice [24-26]. Importantly, reduced HDAC4 expression and increased nuclear shuttling are detected in ischemic stroke model cells and animals, while multiple HDACs, including HDAC2, are increased in ischemic stroke models [9-11, 17, 21, 64]. Moreover, increased HDAC4 expression reduces infarct volume in ischemic stroke model animals and increases cell viability of OGD-treated neurons, while reduced HDAC2 expression promotes neuronal survival and functional recovery in ischemic stroke model animals $[9,10,21,22]$. Consistently, pan-HDACs inhibitors and the specific inhibitor of class I HDACs, including HDAC2, alleviate stroke-induced neurological deficits facilitating poststroke recovery in mice. However, the specific class IIa inhibitor increases mortality and infarct volume in the brains of ischemic stroke model rats, exacerbates neuronal remodeling impairment, and has no rescue effect on neurological deficits $[21,22,65]$.
The alteration and function of HDAC4 are opposite to those of HDAC2 in ischemic stroke models, indicating that increasing HDAC4 expression is a unique target for the treatment of ischemic stroke compared with inhibiting HDAC2 and other HDACs to treat ischemic stroke. Although it is inconclusive that increasing HDAC4 expression could offer a better ischemic stroke therapy compared with HDAC2 inhibition, co-regulating HDAC4 and HDAC2 or other HDACs might have better therapeutic potential. The combination effect of increasing the HDAC4 level and inhibiting the activity of HDAC2 or other HDACs needs to be further investigated.

\section{Current status of HDACs-based treatment}

Currently, thrombolysis with tissue plasminogen activator remains the only globally approved treatment for ischemic stroke [5]. No HDAC-based approach or agent has been approved for ischemic stroke treatment, although four pan-HDAC inhibitors, vorinostat, romidepsin, belinostat, and panobinostat, are approved by the US FDA for the treatment of cutaneous $\mathrm{T}$ cell lymphoma, peripheral $\mathrm{T}$ cell lymphoma, and multiple myeloma, respectively [86, 87]. More than 350 clinical trials involving HDAC inhibitors (https://www.clinicaltrials.gov/) have been carried out or are ongoing against various diseases, including cancers, Alzheimer's disease, schizophrenia, asthma, and chronic obstructive pulmonary disease (COPD). However, no HDAC-based clinical trial has been carried out for ischemic stroke. Moreover, it still lacks HDAC4-based preclinical studies on larger animals, although the therapeutic effect of HDAC4 has been observed in neurons, rats, and mice. Therefore, further investigation is needed before HDAC4-based clinical trials.

\section{Potential of HDAC4-based therapy for ischemic stroke}

Accumulated evidence suggested that increasing HDAC4 expression may have therapeutic potential for ischemic stroke treatment. Several approaches of regulating HDAC4 level could be translated into the clinic (Fig. 2). Adenovirusand adeno-associated virus-mediated HDAC4 overexpression has been applied in vitro and in vivo, indicating that virus-based HDAC4 overexpression could be a potential gene therapy for ischemic stroke treatment [16, 88-91]. However, further preclinical investigation is needed to determine the therapeutic effect on larger animals other than rodents. In addition, the efficacy and safety need to be evaluated.

MicroRNA-based therapies hold great promise in various diseases. Significant alteration of microRNAs targeting HDAC4 has been detected in ischemic stroke patients and model animals, indicating that modulating microRNAs targeting HDAC4 could be a therapeutic approach. A number of microRNAs targeting HDAC4 


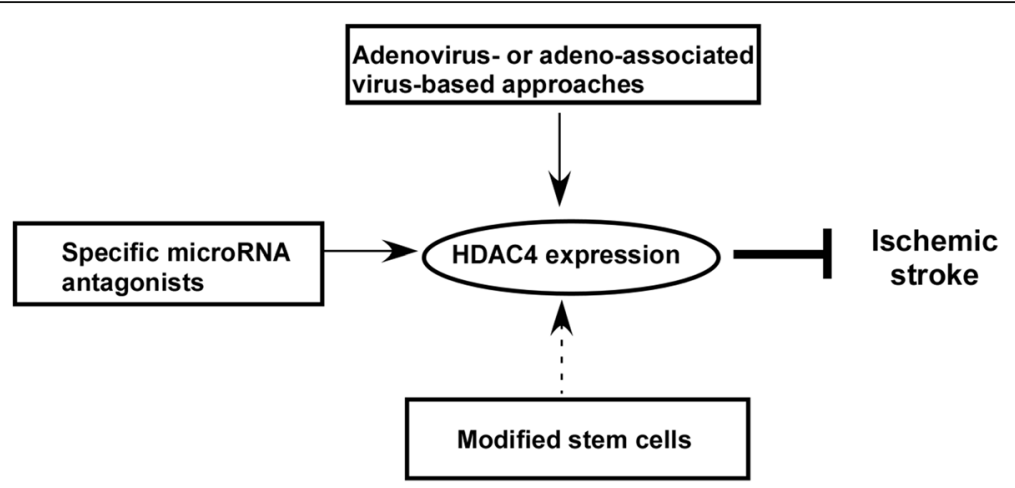

Fig. 2 Potential of HDAC4-based therapy for ischemic stroke. Adenovirus- or adeno-associated virus-mediated HDAC4 overexpression and microRNA-based upregulation of HDAC4 have the potential to be translated into the clinic for ischemic stroke treatment. The therapeutic potential of HDAC4-modified stem cells remains elusive

are increased, e.g., miR-9, miR-124, miR-29b, and miR-206, suggesting that restoring or downregulating their levels may subsequently increase HDAC4 expression. Specific microRNA antagonists, including antimiRs, locked nucleic acids, and antagomirs, could restore HDAC4 expression or increase HDAC4 expressions. Among them, antagomirs can be delivered without any vector or vehicle assistance. A recent study showed that intranasal administration of an antagomir specifically targeting miR-206 significantly improved memory function in the model mice of Alzheimer's disease [92]. It suggested that the non-invasive intranasal administration of specific antagomirs could be an effective approach to increase HDAC4 expression for ischemic stroke treatment. Further preclinical investigation needs to be done to determine the specificity, efficacy, and safety of this approach. The combination effect of targeting various microRNAs needs to be investigated.

Both preclinical studies and clinical trials indicated that stem cell-based therapies would be an effective approach for the treatment of many kinds of diseases, including ischemic stroke [93, 94]. In addition to numerous preclinical studies, a variety of stem cell-based clinical trials for the treatment of ischemic stroke have been carried out or are ongoing, including neural stem cells, mesenchymal stem cells, embryonic stem cells, and induced pluripotent stem cells (https://www.clinicaltrials.gov/). For example, the consistent efficacy of neural stem cell transplantation for ischemic stroke treatment was observed in both preclinical studies and clinical trials, e.g., the trial of Pilot Investigation of Human Neural Stem Cells in Chronic Ischemic Stroke Patients (PISCES) [40, 41]. The results of the PISCES trial might be more conclusive with the enrolment of additional patients and the introduction of a placebo control group in the phase 2 trial (NCT02117635) [40]. Whether HDAC4-modified stem cells could have a better therapeutic effect in patients with ischemic stroke needs to be further investigated. First, the alteration of HDAC4 in different types of stem cells is unclear as only one report showed that both total HDAC4 and cytoplasmic HDAC4 was increased in oligodendrocyte progenitor cells of ischemic stroke model rats [56]. In addition, the role of HDAC4 in different types of stem cells and underlying mechanisms remain elusive.

\section{Conclusions}

HDAC4 expression was reduced in ischemic stroke, which may contribute to the pathogenesis of ischemic stroke by promoting neuronal death and inhibiting angiogenesis and neurogenesis. The increased HDAC4 expression could inhibit neuronal death via reducing HMGB1 expression and release and promote angiogenesis and neurogenesis via HIF- $1 \alpha-\mathrm{VEGF}$ signaling and CREBBDBF signaling, respectively. The interacting partners of HDAC4, MEF2, Runx2, SRF, HP1, ATF4, and NF-kB might also mediate its role in inhibiting neuronal death and promoting angiogenesis and neurogenesis in ischemic stroke. Importantly, it remains to find similar pattern and mechanisms in patients with ischemic stroke as most studies are performed in cultured neurons and animal models. Currently, a number of approaches to regulate HDAC4 level have the potential to be translated into the clinic, such as adenovirus-/adeno-associated virus-mediated HDAC4 overexpression and microRNAbased upregulation of HDAC4. Although a variety of stem cell-based clinical trials for the treatment of ischemic stroke has been carried out or are ongoing, the therapeutic potential of HDAC4-modified stem cells remains elusive. Therefore, modulating HDAC4 expression could be translated into the clinic as an effective treatment for ischemic stroke. However, the therapeutic potential of HDAC4-modified stem cells needs to be further investigated in preclinical studies. 


\section{Abbreviations}

NF-kB: Nuclear factor kappa B; 14-3-3: Andtyrosine 3-monooxygenase/tryptophan 5-monooxygenase activation protein; BBB: Blood-brain barrier; BDNF: Brain-derived neurotrophic factor; CaMK IV: Calcium/calmodulin-dependent protein kinase IV; CaMKII: Calcium/calmodulin-dependent kinase II; CREB: CAMP response element-binding protein; CSF: Cerebrospinal fluid; ER: Endoplasmic reticulum; HDAC: Histone deacetylase; HP1: Heterochromatin protein 1; MCAO: Middle cerebral artery occlusion; MEF2: Myocyte enhancer factor 2; NADPH: Nicotinamide adenine dinucleotide phosphate-oxidase; NMDA: Nmethyl-D-aspartic acid; OGD: Oxygen-glucose deprivation; PKC: Protein kinase $C_{i}$ PP2A: Protein phosphotase 2A; Runx2: Runt-related transcription factor 2; SIRT1: Sirtuin 1; SRF: Serum response factor

\section{Funding}

The present work was funded by the National Natural Science Foundation of China (81771147), Natural Science Foundation of Shandong Province (ZR2016HM30), Young Teachers Research Support Foundation of Jining Medical University (JY2016KJ028Y), and Teachers Research Support Foundation of Jining Medical University (JY2017JS001).

\section{Authors' contributions}

$\mathrm{QK}, \mathrm{YH}, \mathrm{XL}$, and XW wrote the manuscript. BJ wrote and revised the manuscript. YW formulated and revised the manuscript. All authors read and approved the final manuscript

\section{Competing interests}

The authors declare that they have no competing interests.

\section{Publisher's Note}

Springer Nature remains neutral with regard to jurisdictional claims in published maps and institutional affiliations.

\section{Author details}

${ }^{1}$ Collaborative Innovation Center for Birth Defect Research and Transformation of Shandong Province, Jining Medical University, 133 Hehua Road, Taibaihu New District, Jining 272067, Shandong, China. ${ }^{2}$ Department of Biochemistry, Jining Medical University, 133 Hehua Road, Taibaihu New District, Jining 272067, Shandong, China. ${ }^{3}$ Department of Neurology, Affiliated Hospital of Jining Medical University, 89 Guhuai Road, Jining, Jining 272000, Shandong, China. ${ }^{4}$ TEDA international Cardiovascular Hospital, 61 3rd AV, TEDA, Tianjin, China. ${ }^{5}$ Institute of Mental Health, Jining Medical University, 133 Hehua Road, Taibaihu New District, Jining 272067, Shandong, China. ${ }^{6}$ Department of Psychiatry, Jining Medical University, 133 Hehua Road, Taibaihu New District, Jining 272067, Shandong, China.

Received: 11 May 2018 Accepted: 28 August 2018

Published online: 12 September 2018

\section{References}

1. Wang R, Ying Z, Zhao J, Zhang Y, Lu H, Deng Y, et al. Lys(203) and Lys(382) are essential for the proteasomal degradation of BACE1. Curr Alzheimer Res. 2012:9(5):606-15.

2. Mozaffarian D, Benjamin EJ, Go AS, Arnett DK, Blaha MJ, Cushman M, et al. Heart Disease and Stroke Statistics-2016 update: a report from the American Heart Association. Circulation. 2016;133(4):e38-360

3. Wang H, Naghavi M, Allen C, Barber RM, Bhutta ZA, Carter A, et al. Global, regional, and national life expectancy, all-cause mortality, and cause-specific mortality for 249 causes of death, 1980-2015: a systematic analysis for the Global Burden of Disease Study 2015. Lancet. 2016;388(10053):1459-544.

4. Wu Y, Xu Q, Song W. Oxidative stress and Alzheimer's disease. In: Laher I, editor. Systems biology of free radicals and antioxidants. Berlin: Springer Berlin Heidelberg; 2014. p. 2147-74.

5. Chapman SN, Mehndiratta P, Johansen MC, MCMurry TL, Johnston KC, Southerland AM. Current perspectives on the use of intravenous recombinant tissue plasminogen activator (tPA) for treatment of acute ischemic stroke. Vasc Health Risk Manag. 2014;10:75-87. https://doi.org/10. 2147NHRM.S39213.

6. Lardenoije R, latrou A, Kenis G, Kompotis K, Steinbusch HW, Mastroeni D, et al. The epigenetics of aging and neurodegeneration. Prog Neurobiol. 2015; 131:21-64. https://doi.org/10.1016/j.pneurobio.2015.05.002.
7. Han X, Niu J, Zhao Y, Kong Q, Tong T, Han L. HDAC4 stabilizes SIRT1 via sumoylation SIRT1 to delay cellular senescence. Clin Exp Pharmacol Physiol. 2016;43(1):41-6. https://doi.org/10.1111/1440-1681.12496.

8. Hohl M, Wagner M, Reil JC, Muller SA, Tauchnitz M, Zimmer AM, et al. HDAC4 controls histone methylation in response to elevated cardiac load. J Clin Invest. 2013;123(3):1359-70. https://doi.org/10.1172/JCl61084.

9. He M, Zhang B, Wei X, Wang Z, Fan B, Du P, et al. HDAC4/5-HMGB1 signalling mediated by NADPH oxidase activity contributes to cerebral ischaemia/reperfusion injury. J Cell Mol Med. 2013;17(4):531-42. https://doi. org/10.1111/jcmm.12040.

10. Zhang QY, Wang ZJ, Sun DM, Wang Y, Xu P, Wu WJ, et al. Novel therapeutic effects of leonurine on ischemic stroke: new mechanisms of BBB integrity. Oxidative Med Cell Longev. 2017;2017:7150376. https://doi.org/10.1155/ 2017/7150376

11. Yuan H, Denton K, Liu L, Li XJ, Benashski S, McCullough L, et al. Nuclear translocation of histone deacetylase 4 induces neuronal death in stroke. Neurobiol Dis. 2016;91:182-93. https://doi.org/10.1016/j.nbd.2016.03.004

12. Morris B, Etoubleau C, Bourthoumieu S, Reynaud-Perrine S, Laroche C, Lebbar A, et al. Dose dependent expression of HDAC4 causes variable expressivity in a novel inherited case of brachydactyly mental retardation syndrome. Am J Med Genet A. 2012;158A(8):2015-20. https://doi.org/10. 1002/ajmg.a.35463.

13. Otsuki K, Uchida S, Hobara T, Yamagata H, Watanabe Y. Epigenetic regulation in depression. Nihon Shinkei Seishin Yakurigaku Zasshi. 2012; 32(4):181-6.

14. Shen X, Chen J, Li J, Kofler J, Herrup K. Neurons in vulnerable regions of the Alzheimer's disease brain display reduced ATM signaling. eNeuro. 2016:3:1. https://doi.org/10.1523/ENEURO.0124-15.2016.

15. Wu Y, Hou F, Wang X, Kong Q, Han X, Bai B. Aberrant expression of histone deacetylases 4 in cognitive disorders: molecular mechanisms and a potential target. Front Mol Neurosci. 2016;9:114. https://doi.org/10.3389/ fnmol.2016.00114

16. Majdzadeh N, Wang L, Morrison BE, Bassel-Duby R, Olson EN, D'Mello SR. HDAC4 inhibits cell-cycle progression and protects neurons from cell death. Dev Neurobiol. 2008;68(8):1076-92. https://doi.org/10.1002/dneu.20637.

17. Chen YT, Zang XF, Pan J, Zhu XL, Chen F, Chen ZB, et al. Expression patterns of histone deacetylases in experimental stroke and potential targets for neuroprotection. Clin Exp Pharmacol Physiol. 2012;39(9):751-8. https://doi.org/10.1111/j.1440-1681.2012.05729.x.

18. Sen T, Sen N. Isoflurane-induced inactivation of CREB through histone deacetylase 4 is responsible for cognitive impairment in developing brain. Neurobiol Dis. 2016;96:12-21. https://doi.org/10.1016/j.nbd.2016.08.005.

19. Zhang G, Zhang T, Li N, Wu L, Gu J, Li C, et al. Tetramethylpyrazine nitrone activates the BDNF/Akt/CREB pathway to promote post-ischaemic neuroregeneration and recovery of neurological functions in rats. $\mathrm{Br} \mathrm{J}$ Pharmacol. 2018;175(3):517-31. https://doi.org/10.1111/bph.14102.

20. Jeong $\mathrm{CH}$, Kim SM, Lim JY, Ryu CH, Jun JA, Jeun SS. Mesenchymal stem cells expressing brain-derived neurotrophic factor enhance endogenous neurogenesis in an ischemic stroke model. Biomed Res Int. 2014;2014 129145. https://doi.org/10.1155/2014/129145.

21. Tang Y, Lin YH, Ni HY, Dong J, Yuan HJ, Zhang Y, et al. Inhibiting histone deacetylase 2 (HDAC2) promotes functional recovery from stroke. J Am Heart Assoc. 2017;6:10. https://doi.org/10.1161/JAHA.117.007236.

22. Lin YH, Dong J, Tang Y, Ni HY, Zhang Y, Su P, et al. Opening a new time window for treatment of stroke by targeting HDAC2. J Neurosci. 2017. 37(28):6712-28. https://doi.org/10.1523/JNEUROSCI.0341-17.2017.

23. Han Z, Dong X, Zhang C, Wu Y, Yuan Z, Wang X. Polymorphism of HDAC9 gene is associated with increased risk of acute coronary syndrome in Chinese Han population. Biomed Res Int. 2016;2016:3746276. https://doi. org/10.1155/2016/3746276

24. Vecsey CG, Hawk JD, Lattal KM, Stein JM, Fabian SA, Attner MA, et al. Histone deacetylase inhibitors enhance memory and synaptic plasticity via CREB: CBP-dependent transcriptional activation. J Neurosci. 2007;27(23): 6128-40. https://doi.org/10.1523/JNEUROSCl.0296-07.2007.

25. Guan JS, Haggarty SJ, Giacometti E, Dannenberg JH, Joseph N, Gao J, et al. HDAC2 negatively regulates memory formation and synaptic plasticity. Nature. 2009;459(7243):55-60. https://doi.org/10.1038/nature07925.

26. Kim MS, Akhtar MW, Adachi M, Mahgoub M, Bassel-Duby R, Kavalali ET, et al. An essential role for histone deacetylase 4 in synaptic plasticity and memory formation. J Neurosci. 2012:32(32):10879-86. https://doi.org/10. 1523/JNEUROSCI.2089-12.2012. 
27. Wu Y, Wang X, Zhou X, Cheng B, Li G, Bai B. Temporal expression of Apelin/ Apelin receptor in ischemic stroke and its therapeutic potential. Front Mol Neurosci. 2017;10:1. https://doi.org/10.3389/fnmol.2017.00001.

28. Moskowitz MA, Lo EH, ladecola C. The science of stroke: mechanisms in search of treatments. Neuron. 2010;67(2):181-98. https://doi.org/10.1016/j. neuron.2010.07.002.

29. Zhang S, Zis O, Ly PT, Wu Y, Zhang M, Cai F, et al. Down-regulation of MIF by NFkappaB under hypoxia accelerated neuronal loss during stroke. FASEB J. 2014; https://doi.org/10.1096/fj.14-253625.

30. Gutierrez-Vargas JA, Munoz-Manco Jl, Garcia-Segura LM, Cardona-Gomez GP. GluN2B N-methyl-D-aspartic acid receptor subunit mediates atorvastatin-induced neuroprotection after focal cerebral ischemia. J Neurosci Res. 2014;92(11):1529-48. https://doi.org/10.1002/jnr.23426.

31. Krupinski J, Kaluza J, Kumar P, Kumar S, Wang JM. Role of angiogenesis in patients with cerebral ischemic stroke. Stroke. 1994;25(9):1794-8.

32. Arenillas JF, Sobrino T, Castillo J, Davalos A. The role of angiogenesis in damage and recovery from ischemic stroke. Curr Treat Options Cardiovasc Med. 2007;9(3):205-12.

33. Zhang ZG, Chopp M. Promoting brain remodeling to aid in stroke recovery. Trends Mol Med. 2015;21(9):543-8. https://doi.org/10.1016/j.molmed. 2015.07.005.

34. Zhu J, Liu Q, Jiang Y, Wu L, Xu G, Liu X. Enhanced angiogenesis promoted by human umbilical mesenchymal stem cell transplantation in stroked mouse is Notch1 signaling associated. Neuroscience. 2015;290:288-99. https://doi.org/10.1016/j.neuroscience.2015.01.038.

35. Seto SW, Chang D, Jenkins A, Bensoussan A, Kiat H. Angiogenesis in ischemic stroke and angiogenic effects of Chinese herbal medicine. J Clin Med. 2016;5:6. https:/doi.org/10.3390/jcm5060056.

36. Li Y, Zhang X, Cui L, Chen R, Zhang Y, Zhang C, et al. Salvianolic acids enhance cerebral angiogenesis and neurological recovery by activating JAK2/STAT3 signaling pathway after ischemic stroke in mice. J Neurochem. 2017; https://doi.org/10.1111/jnc.14140.

37. Ruan L, Wang B, ZhuGe Q, Jin K. Coupling of neurogenesis and angiogenesis after ischemic stroke. Brain Res. 2015;1623:166-73. https://doi. org/10.1016/j.brainres.2015.02.042

38. Lindvall O, Kokaia Z. Neurogenesis following stroke affecting the adult brain. Cold Spring Harb Perspect Biol. 2015;7:11. https://doi.org/10.1101/ cshperspect.a019034

39. Guo F, Lou J, Han X, Deng Y, Huang X. Repetitive transcranial magnetic stimulation ameliorates cognitive impairment by enhancing neurogenesis and suppressing apoptosis in the hippocampus in rats with ischemic stroke. Front Physiol. 2017;8:559. https://doi.org/10.3389/fphys.2017.00559.

40. Borlongan CV. Age of PISCES: stem-cell clinical trials in stroke. Lancet. 2016; 388(10046):736-8. https://doi.org/10.1016/50140-6736(16)31259-4.

41. Lu J, Manaenko A, Hu Q. Targeting adult neurogenesis for poststroke therapy. Stem Cells Int. 2017;2017:5868632. https://doi.org/10.1155/2017/5868632.

42. Nagpal A, Choy FC, Howell S, Hillier S, Chan F, Hamilton-Bruce MA, et al. Safety and effectiveness of stem cell therapies in early-phase clinical trials in stroke: a systematic review and meta-analysis. Stem Cell Res Ther. 2017;8(1): 191. https://doi.org/10.1186/s13287-017-0643-X.

43. Mielcarek M, Landles C, Weiss A, Bradaia A, Seredenina T, Inuabasi L, et al. HDAC4 reduction: a novel therapeutic strategy to target cytoplasmic huntingtin and ameliorate neurodegeneration. PLoS Biol. 2013;11(11): e1001717. https://doi.org/10.1371/journal.pbio.1001717.

44. Nishino TG, Miyazaki M, Hoshino H, Miwa Y, Horinouchi S, Yoshida M. 14-3-3 regulates the nuclear import of class lla histone deacetylases. Biochem Biophys Res Commun. 2008;377(3):852-6. https://doi.org/10.1016/j.bbrc.2008.10.079.

45. Wu Q, Yang X, Zhang L, Zhang Y, Feng L. Nuclear accumulation of histone deacetylase 4 (HDAC4) exerts neurotoxicity in models of Parkinson's disease. Mol Neurobiol. 2016; https://doi.org/10.1007/s12035-016-0199-2.

46. Litke C, Bading H, Mauceri D. Histone deacetylase 4 shapes neuronal morphology via a mechanism involving regulation of expression of vascular endothelial growth factor D. J Biol Chem. 2018;293(21):8196-207. https:// doi.org/10.1074/jbc.RA117.001613.

47. Wang Z, Qin G, Zhao TC. HDAC4: mechanism of regulation and biological functions. Epigenomics. 2014;6(1):139-50. https://doi.org/10.2217/epi.13.73.

48. Grozinger CM, Hassig CA, Schreiber SL. Three proteins define a class of human histone deacetylases related to yeast Hda1p. Proc Natl Acad Sci U S A. 1999;96(9):4868-73.

49. Lee HA, Song MJ, Seok YM, Kang SH, Kim SY, Kim I. Histone deacetylase 3 and 4 complex stimulates the transcriptional activity of the mineralocorticoid receptor. PLoS One. 2015;10(8):e0136801. https://doi.org/ 10.1371/journal.pone.0136801.

50. Ginnan R, Sun LY, Schwarz JJ, Singer HA. MEF2 is regulated by CaMKIldelta2 and a HDAC4-HDAC5 heterodimer in vascular smooth muscle cells. Biochem J. 2012;444(1):105-14. https://doi.org/10.1042/BJ20120152.

51. Sando R 3rd, Gounko N, Pieraut S, Liao L, Yates J 3rd, Maximov A. HDAC4 governs a transcriptional program essential for synaptic plasticity and memory. Cell. 2012;151(4):821-34. https://doi.org/10.1016/j.cell.2012.09.037.

52. Ronan JL, Wu W, Crabtree GR. From neural development to cognition: unexpected roles for chromatin. Nat Rev Genet. 2013;14(5):347-59. https:// doi.org/10.1038/nrg3413.

53. Zhang $P$, Sun $Q$, Zhao C, Ling S, Li Q, Chang YZ, et al. HDAC4 protects cells from ER stress induced apoptosis through interaction with ATF4. Cell Signal. 2014;26(3):556-63. https://doi.org/10.1016/j.cellsig.2013.11.026.

54. Schwartz S, Truglio M, Scott MJ, Fitzsimons HL. Long-term memory in Drosophila is influenced by the histone deacetylase HDAC4 interacting with the SUMO-conjugating enzyme Ubc9. Genetics. 2016; https://doi.org/10. 1534/genetics.115.183194.

55. Kang B, Li W, Xi W, Yi Y, Ciren Y, Shen H, et al. Hydrogen sulfide protects cardiomyocytes against apoptosis in ischemia/reperfusion through MiR-1regulated histone deacetylase 4 pathway. Cell Physiol Biochem. 2017:41(1): 10-21. https://doi.org/10.1159/000455816.

56. Kassis H, Chopp M, Liu XS, Shehadah A, Roberts C, Zhang ZG. Histone deacetylase expression in white matter oligodendrocytes after stroke. Neurochem Int. 2014;77:17-23. https://doi.org/10.1016/j.neuint.2014.03.006.

57. Sorensen SS, Nygaard AB, Carlsen AL, Heegaard NHH, Bak M, Christensen T. Elevation of brain-enriched miRNAs in cerebrospinal fluid of patients with acute ischemic stroke. Biomark Res. 2017;5:24. https://doi.org/10.1186/ s40364-017-0104-9.

58. Ji Q, Ji Y, Peng J, Zhou X, Chen X, Zhao H, et al. Increased brain-specific MiR-9 and MiR-124 in the serum exosomes of acute ischemic stroke patients. PLoS One. 2016;11(9):e0163645. https://doi.org/10.1371/journal. pone. 0163645

59. Liu Y, Zhang J, Han R, Liu H, Sun D, Liu X. Downregulation of serum brain specific microRNA is associated with inflammation and infarct volume in acute ischemic stroke. J Clin Neurosci. 2015;22(2):291-5. https://doi.org/10. 1016/j.jocn.2014.05.042.

60. Wei N, Xiao L, Xue R, Zhang D, Zhou J, Ren $\mathrm{H}$, et al. MicroRNA-9 mediates the cell apoptosis by targeting Bcl2l11 in ischemic stroke. Mol Neurobiol. 2016;53(10):6809-17. https://doi.org/10.1007/s12035-015-9605-4.

61. Shi G, Liu Y, Liu T, Yan W, Liu X, Wang Y, et al. Upregulated miR-29b promotes neuronal cell death by inhibiting Bcl2L2 after ischemic brain injury. Exp Brain Res. 2012;216(2):225-30. https://doi.org/10.1007/s00221011-2925-3.

62. Winbanks CE, Wang B, Beyer C, Koh P, White L, Kantharidis P, et al. TGF-beta regulates miR-206 and miR-29 to control myogenic differentiation through regulation of HDAC4. J Biol Chem. 2011;286(16):13805-14. https://doi.org/10 1074/jbc.M110.192625.

63. Liu FJ, Lim KY, Kaur P, Sepramaniam S, Armugam A, Wong PT, et al. microRNAs involved in regulating spontaneous recovery in embolic stroke model. PLoS One. 2013;8(6):e66393. https:/doi.org/10.1371/journal.pone.0066393.

64. Kassis H, Shehadah A, Chopp M, Roberts C, Zhang ZG. Stroke induces nuclear shuttling of histone deacetylase 4. Stroke. 2015;46(7):1909-15. https://doi.org/10.1161/STROKEAHA.115.009046.

65. Kassis H, Shehadah A, Li C, Zhang Y, Cui Y, Roberts C, et al. Class lla histone deacetylases affect neuronal remodeling and functional outcome after stroke. Neurochem Int. 2016;96:24-31. https://doi.org/10.1016/j.neuint.2016.04.006.

66. Liu XS, Chopp M, Kassis H, Jia LF, Hozeska-Solgot A, Zhang RL, et al. Valproic acid increases white matter repair and neurogenesis after stroke. Neuroscience. 2012;220:313-21. https://doi.org/10.1016/j.neuroscience.2012.06.012.

67. Baltan S, Murphy SP, Danilov CA, Bachleda A, Morrison RS. Histone deacetylase inhibitors preserve white matter structure and function during ischemia by conserving ATP and reducing excitotoxicity. J Neurosci. 2011;31(11):3990-9. https://doi.org/10.1523/JNEUROSCI.5379-10.2011.

68. Neuner SM, Wilmott LA, Hoffmann BR, Mozhui K, Kaczorowski CC. Hippocampal proteomics defines pathways associated with memory decline and resilience in 'normal' aging and Alzheimer's disease mouse models. Behav Brain Res. 2016; https://doi.org/10.1016/.bbr.2016.06.002.

69. Fitzsimons HL, Schwartz S, Given FM, Scott MJ. The histone deacetylase HDAC4 regulates long-term memory in Drosophila. PLoS One. 2013;8(12): e83903. https://doi.org/10.1371/journal.pone.0083903. 
70. Luan B, Goodarzi MO, Phillips NG, Guo X, Chen YD, Yao J, et al. Leptinmediated increases in catecholamine signaling reduce adipose tissue inflammation via activation of macrophage HDAC4. Cell Metab. 2014;19(6): 1058-65. https://doi.org/10.1016/j.cmet.2014.03.024.

71. Yang Y, Qin X, Liu S, Li J, Zhu X, Gao T, et al. Peroxisome proliferatoractivated receptor gamma is inhibited by histone deacetylase 4 in cortical neurons under oxidative stress. J Neurochem. 2011;118(3):429-39. https:// doi.org/10.1111/j.1471-4159.2011.07316.x.

72. Wu Q, Yang X, Zhang L, Zhang Y, Feng L. Nuclear accumulation of histone deacetylase 4 (HDAC4) exerts neurotoxicity in models of parkinson's disease. Mol Neurobiol. 2017:54(9):6970-83. https://doi.org/10.1007/s12035-016-0199-2.

73. Qian DZ, Kachhap SK, Collis SJ, Verheul HM, Carducci MA, Atadja P, et al. Class II histone deacetylases are associated with VHL-independent regulation of hypoxia-inducible factor 1 alpha. Cancer Res. 2006;66(17): 8814-21. https://doi.org/10.1158/0008-5472.CAN-05-4598.

74. Granger A, Abdullah I, Huebner F, Stout A, Wang T, Huebner T, et al. Histone deacetylase inhibition reduces myocardial ischemia-reperfusion injury in mice. FASEB J. 2008;22(10):3549-60. https://doi.org/10.1096/fj.08-108548.

75. Liu J, Zhou X, Li Q, Zhou SM, Hu B, Hu GW, et al. Role of phosphorylated HDAC4 in stroke-induced angiogenesis. Biomed Res Int. 2017;2017:2957538. https://doi.org/10.1155/2017/2957538.

76. Xu Q, Liu LZ, Yin Y, He J, Li Q, Qian X, et al. Regulatory circuit of PKM2/NFkappaB/miR-148a/152-modulated tumor angiogenesis and cancer progression. Oncogene. 2015;34(43):5482-93. https://doi.org/10.1038/onc.2015.6.

77. Madelaine R, Sloan SA, Huber N, Notwell JH, Leung LC, Skariah G, et al. MicroRNA-9 couples brain neurogenesis and angiogenesis. Cell Rep. 2017; 20(7):1533-42. https://doi.org/10.1016/j.celrep.2017.07.051.

78. Jiang C, Zuo F, Wang Y, Lu H, Yang Q, Wang J. Progesterone changes VEGF and BDNF expression and promotes neurogenesis after ischemic stroke. Mol Neurobiol. 2016. Epub ahead of print. https://doi.org/10.1007/s12035-0159651-y.

79. Davila JL, Goff LA, Ricupero CL, Camarillo C, Oni EN, Swerdel MR, et al. A positive feedback mechanism that regulates expression of miR-9 during neurogenesis. PLoS One. 2014;9(4):e94348. https://doi.org/10.1371/journal. pone.0094348.

80. Latchney SE, Jiang Y, Petrik DP, Eisch AJ, Hsieh J. Inducible knockout of Mef2a, - $c$, and - $d$ from nestin-expressing stem/progenitor cells and their progeny unexpectedly uncouples neurogenesis and dendritogenesis in vivo. FASEB J. 2015;29(12):5059-71. https://doi.org/10.1096/fj.15-275651.

81. Li Z, McKercher SR, Cui J, Nie Z, Soussou W, Roberts AJ, et al. Myocyte enhancer factor $2 \mathrm{C}$ as a neurogenic and antiapoptotic transcription factor in murine embryonic stem cells. J Neurosci. 2008;28(26):6557-68. https://doi. org/10.1523/JNEUROSCI.0134-08.2008.

82. Wang AH, Yang XJ. Histone deacetylase 4 possesses intrinsic nuclear import and export signals. Mol Cell Biol. 2001;21(17):5992-6005.

83. McKinsey TA, Zhang CL, Lu J, Olson EN. Signal-dependent nuclear export of a histone deacetylase regulates muscle differentiation. Nature. 2000; 408(6808):106-11. https://doi.org/10.1038/35040593.

84. McKinsey TA, Zhang CL, Olson EN. Identification of a signal-responsive nuclear export sequence in class II histone deacetylases. Mol Cell Biol. 2001; 21(18):6312-21.

85. Lahm A, Paolini C, Pallaoro M, Nardi MC, Jones P, Neddermann P, et al. Unraveling the hidden catalytic activity of vertebrate class lla histone deacetylases. Proc Natl Acad Sci U S A. 2007;104(44):17335-40. https://doi. org/10.1073/pnas.0706487104.

86. Falkenberg KJ, Johnstone RW. Histone deacetylases and their inhibitors in cancer, neurological diseases and immune disorders. Nat Rev Drug Discov. 2014;13(9):673-91. https://doi.org/10.1038/nrd4360.

87. Faria Freitas M, Cuendet M, Bertrand P. HDAC inhibitors: a 2013-2017 patent survey. Expert Opin Ther Pat. 2018:1-17. https://doi.org/10.1080/ 13543776.2018.1459568.

88. Gaur V, Connor T, Sanigorski A, Martin SD, Bruce CR, Henstridge DC, et al. Disruption of the class lla HDAC corepressor complex increases energy expenditure and lipid oxidation. Cell Rep. 2016;16(11):2802-10. https://doi. org/10.1016/j.celrep.2016.08.005.

89. Zhang LX, DeNicola M, Qin X, Du J, Ma J, Tina Zhao Y, et al. Specific inhibition of HDAC4 in cardiac progenitor cells enhances myocardial repairs. Am J Physiol Cell Physiol. 2014;307(4):C358-72. https://doi.org/10.1152/ ajpcell.00187.2013.

90. Khalil W, Xia H, Bodempudi V, Kahm J, Hergert P, Smith K, et al. Pathologic regulation of collagen I by an aberrant protein phosphatase 2A/histone deacetylase C4/MicroRNA-29 signal axis in idiopathic pulmonary fibrosis fibroblasts. Am J Respir Cell Mol Biol. 2015;53(3):391-9. https:/doi.org/10. 1165/rcmb.2014-01500C

91. Chen C, Wei X, Wang S, Jiao Q, Zhang Y, Du G, et al. Compression regulates gene expression of chondrocytes through HDAC4 nuclear relocation via PP2A-dependent HDAC4 dephosphorylation. Biochim Biophys Acta. 2016; 1863(7 Pt A):1633-42. https://doi.org/10.1016/j.bbamcr.2016.04.018.

92. Lee ST, Chu K, Jung KH, Kim JH, Huh JY, Yoon H, et al. miR-206 regulates brain-derived neurotrophic factor in Alzheimer disease model. Ann Neurol. 2012;72(2):269-77. https://doi.org/10.1002/ana.23588.

93. Marei HE, Hasan A, Rizzi R, Althani A, Afifi N, Cenciarelli C, et al. Potential of stem cell-based therapy for ischemic stroke. Front Neurol. 2018;9:34. https:// doi.org/10.3389/fneur.2018.00034.

94. Trounson A, McDonald C. Stem cell therapies in clinical trials: progress and challenges. Cell Stem Cell. 2015;17(1):11-22. https://doi.org/10.1016/j.stem. 2015.06.007.

\section{Ready to submit your research? Choose BMC and benefit from:}

- fast, convenient online submission

- thorough peer review by experienced researchers in your field

- rapid publication on acceptance

- support for research data, including large and complex data types

- gold Open Access which fosters wider collaboration and increased citations

- maximum visibility for your research: over $100 \mathrm{M}$ website views per year

At $\mathrm{BMC}$, research is always in progress.

Learn more biomedcentral.com/submissions 